\title{
SHADOWING PROPERTY OF CONTINUOUS MAPS WITH ZERO TOPOLOGICAL ENTROPY
}

\author{
MILAN KUCHTA
}

(Communicated by Andrew M. Bruckner)

\begin{abstract}
The study of the shadowing property has a long history but for interval maps it is rather new. Recent research in this direction is mainly focused on the positive entropy maps and work for zero entropy is still seldom to be found in the literature. In this paper we give a characterization of zero topological entropy maps which have the shadowing property. Moreover, our condition is necessary for any continuous function to have the shadowing property.
\end{abstract}

\section{INTRODUCTION}

Let $C^{0}(I, I)$ denote the class of continuous maps $I \rightarrow I$, where $I$ is a compact real interval. The orbit (or trajectory) of $x \in I$ with respect to $f$ is the sequence $\operatorname{orb}(x)=\left\{f^{n}(x)\right\}_{n=0}^{\infty}$ where $f^{n}$ denotes the $n$th iterate of $f$. Denote the set of periodic points of $f$ by $\operatorname{Per}(f)$, the set of the fixed points of $f$ by $\operatorname{Fix}(f)$, and the topological entropy of $f$ by $E(f)$. The interval $J \subset I$ is called a periodic interval with period $\operatorname{per}(J)=k \in \mathbb{N}$ if $f^{k}(J)=J$ and $f^{i}(J) \cap f^{j}(J)=\varnothing$ for $0 \leq i \neq j<k$. If $J$ is degenerate to a point then it may be called a periodic point. We will denote a closed interval with $x \leq y$ by $[x, y]$, and a closed interval where no information about order of $x, y$ is provided by $[x, y]^{*}$.

Definition 1.1. If $f \in C^{0}(I, I)$ and $\delta>0$ is given, then a sequence $\mathbf{X}_{\delta}=$ $\left\{\mathbf{x}_{i}\right\}_{i=0}^{\infty}$ of points in $I$ is called a $\delta$-chain of $f$ (or a $\delta$-pseudo orbit of $f$ ) provided that

$$
\left|f\left(\mathbf{x}_{i}\right)-\mathbf{x}_{i+1}\right| \leq \delta \text { for every } i \geq 0 \text {. }
$$

Given $\varepsilon>0$, a $\delta$-chain $\mathbf{X}_{\delta}$ is said to be $\varepsilon$-shadowed by $y \in I$ if

$$
\left|f^{i}(y)-\mathbf{x}_{i}\right| \leq \varepsilon \text { for every } i \geq 0 \text {; }
$$

$f$ is said to have the shadowing property if for any $\varepsilon>0$ there is $\delta>0$ such that every $\delta$-chain of $f$ can be $\varepsilon$-shadowed by a point in $I$.

Definition 1.2. Let $f \in C^{0}(I, I)$. We will call $f$ a shrink function if and only if for every sequence $\left\{J_{k}\right\}_{k=0}^{\infty}$ of periodic intervals such that $J_{k+1} \subset J_{k}$ and $\operatorname{per}\left(J_{k+1}\right)>\operatorname{per}\left(J_{k}\right)$ we have that $\lim _{k \rightarrow \infty}\left|J_{k}\right|=0$.

Received by the editors July 22, 1991 and, in revised form, February 21, 1992.

1991 Mathematics Subject Classification. Primary 54H20, 26A18.

Key words and phrases. Shadowing property, iteration, zero topological entropy. 
Definition 1.3. We will call a one-sided neighborhood $[p, q]^{*}$ of the periodic point $p$ an $m$ - $f$-nontrapping neighborhood of $p$ if $f^{m}(p)=p$ and, for every $x \in[p, q]^{*}, x \in f^{m}\left([p, x]^{*}\right)$. If $m=1$ then we will say that $[p, q]^{*}$ is an $f$-nontrapping neighborhood of $p$.

Definition 1.4. We will call $f \in C^{0}(I, I)$ a nondegenerate function if the following condition holds:

If $x \in I, p \in \operatorname{Per}(f),[p, q]^{*}$ is an $m-f$-nontrapping neighborhood of $p$, and $\lim _{n \rightarrow \infty} f^{m n}(x)=p$, then for every neighborhood $O_{x}$ of $x$ and for all $z_{1}, z_{2} \in(p, q)^{*}$ there is an $n_{0} \in \mathbb{N}$ such that $\left[z_{1}, z_{2}\right]^{*} \subset$ $f^{m n_{0}}\left(O_{x}\right)$.

Main Theorem. Let $f \in C^{0}(I, I)$ and $E(f)=0$. Then $f$ has the shadowing property if and only if $f$ is a nondegenerate shrink function.

Remark 1.5 (cf. Preiss and Smítal [13]). Let $f \in C^{0}(I, I)$ and $E(f)=0$. If $f$ has the shadowing property then $f$ is nonchaotic stable (this means that a perturbed map can be chaotic but thie chaos must be small whenever the perturbation is small).

Remark 1.6. If we use the results from [11], we can easily obtain similar results for continuous maps of the circle.

\section{NECESSARY CONDITIONS}

Let $I$ be a compact interval and $A$ a subset of $I$. Denote by $|A|$ the diameter of $A, \operatorname{int}(A)$ the interior of $A, \operatorname{conv}(A)$ the convex hull of $A$, and $\bar{A}$ the closure of $A$.

First we recall the following well-known fact (see [8]):

Lemma 2.1. Let $f \in C^{0}(I, I)$ and $n \in \mathbb{N}$. Then $f$ has the shadowing property if and only if $f^{n}$ does.

Proposition 2.2. Let $f \in C^{0}(I, I)$. If $f$ is a nonshrink function then it does not have the shadowing property.

Proof. If $f$ is a nonshrink function then there is a sequence $\left\{J_{k}\right\}_{k=0}^{\infty}$ of periodic intervals from Definition 1.2 such that $\lim _{k \rightarrow \infty}\left|J_{k}\right|>0$. Let $J=\bigcap_{k=0}^{\infty} J_{k}$. We have that $J=[p, q]$ where $p \neq q$.

Set $\varepsilon=\frac{|p-q|}{2}$ and $\mathbf{x}_{0}=\frac{p+q}{2}$, and let $\delta>0$. There are $j, k \in \mathbb{N}$ such that

$$
\left|f^{j}\left(J_{k}\right)\right|<\delta,
$$

but there is $\mathbf{x}_{j} \in f^{j}\left(J_{k}\right)$ such that $f^{2^{k}-j}\left(\mathbf{x}_{j}\right)=\mathbf{x}_{0}$. Set

$$
\begin{aligned}
\mathbf{x}_{i} & =f^{i}\left(\mathbf{x}_{0}\right) \text { for } 0<i<j, \\
\mathbf{x}_{j+i} & =f^{i}\left(\mathbf{x}_{j}\right) \text { for } 0<i .
\end{aligned}
$$

We have that $\left\{\mathbf{x}_{i}\right\}_{i=0}^{\infty}$ is a $\delta$-chain (see (1)) and $\mathbf{x}_{0}=\mathbf{x}_{2^{k}}$.

Now if $y \notin J$ then $\left|y-\mathbf{x}_{0}\right|>\varepsilon$ and if $y \in J$ then $\left|f^{2^{k}}(y)-\mathbf{x}_{2^{k}}\right|>\varepsilon$ because $J \cap f^{i}(J)=\varnothing$ for $i \in \mathbb{N}$.

Hence we have found a $\delta$-chain which is not $\varepsilon$-shadowed.

Proposition 2.3. Let $f \in C^{0}(I, I)$ be a degenerate function. Then $f$ does not have the shadowing property. 
Proof. Let $x \in I, \lim _{n \rightarrow \infty} f^{m n}(x)=p,[p, q]^{*}$ be an $m$ - $f$-nontrapping neighborhood of $p$, and let there be $z_{1}, z_{2} \in(p, q)^{*}$ and a neighborhood $O_{x}$ of $x$ such that

$$
\left[z_{1}, z_{2}\right]^{*} \not \subset f^{m n}\left(O_{x}\right) \text { for all } n \in\{0,1, \ldots\} .
$$

Without loss of generality assume that $[p, q]^{*}=[p, q]$ and $z_{1}<z_{2}$. Moreover, we can assume that $m=1$ (see Lemma 2.1).

Let $\varepsilon>0$ such that

$$
\left|q-z_{2}\right|>\varepsilon \text { and }[x-\varepsilon, x+\varepsilon] \subset O_{x} .
$$

Now we claim that for any $\delta>0$ there exists $\mathbf{X}_{\delta}$ which is not $\varepsilon$-shadowed.

There is an $n_{\delta} \in \mathbb{N}$ such that

$$
\begin{gathered}
f^{n}(x)<z_{1} \text { for all } n \geq n_{\delta}, \\
\left|p-f^{n_{\delta}}(x)\right|<\delta .
\end{gathered}
$$

Let $k_{\delta} \in \mathbb{N}$ be such that $k_{\delta}>\frac{|q-p|}{\delta}$. Set $\mathbf{x}_{n_{\delta}+k_{\delta}}=q$, and for $i \in\left\{1, \ldots, k_{\delta}\right\}$ define

$$
\begin{aligned}
\text { if } \quad \mathbf{x}_{n_{\delta}+k_{\delta}-i+1}-\delta \leq p & \text { then } \quad \mathbf{x}_{n_{\delta}+k_{\delta}-i}=p, \\
\text { if } \quad \mathbf{x}_{n_{\delta}+k_{\delta}-i+1}-\delta>p & \text { then } \mathbf{x}_{n_{\delta}+k_{\delta}-i} \in\left[p, \mathbf{x}_{n_{\delta}+k_{\delta}-i+1}-\delta\right] \\
& \text { and } f\left(\mathbf{x}_{n_{\delta}+k_{\delta}-i}\right)=\mathbf{x}_{n_{\delta}+k_{\delta}-i+1}-\delta
\end{aligned}
$$

(this definition is correct because $[p, q]$ is an $f$-nontrapping neighborhood of $p)$, and let $\mathbf{x}_{i}=f^{i}(x)$ for $i \in\left\{0, \ldots, n_{\delta}-1\right\}$.

Hence $\left\{\mathbf{x}_{i}\right\}_{i=0}^{n_{\delta}+k_{\delta}}$ is a $\delta$-chain (see (5)). If $y \notin O_{x}$ then $\left|y-\mathbf{x}_{0}\right|>\varepsilon(\operatorname{see}(3))$ and if $y \in O_{x}$ then $f^{n_{\delta}+k_{\delta}}(y)<z_{2}$ (see (2) and (4)) and so $\left|\mathbf{x}_{n_{\delta}+k_{\delta}}-f^{n_{\delta}+k_{\delta}}(y)\right|>$ $\varepsilon(\operatorname{see}(3))$.

So our $\delta$-chain cannot be $\varepsilon$-shadowed.

Remark 2.4. Note that we do not use the assumption that $E(f)=0$, so Proposition 2.2 and Proposition 2.3 give us a necessary condition for any function from $C^{0}(I, I)$ to have the shadowing property.

\section{COVER SYSTEM}

We begin with

Definition 3.1. Let $f \in C^{0}(I, I)$. We will call $\left\{M_{i}\right\}_{i=0}^{\infty}$ a cover system for $f$ if and only if $M_{i} \subset I$ is a minimal closed set such that $M_{i}=\overline{\bigcup_{k \in K_{i}} I_{k}^{i}}$ where $I_{k}^{i}$ is a closed periodic interval with period $2^{i}$ and, for all $p \in \operatorname{Per}(f), f^{2^{i}}(p) \neq p$ implies $p \in I_{k}^{i}$ for some $k \in K_{i}$.

Lemma 3.2. Let $f \in C^{0}(I, I), E(f)=0, p \in \operatorname{Per}(f)$, and $c \in \operatorname{Fix}(f)$. If $c \notin \operatorname{conv}(\operatorname{orb}(p))$, then $c \notin J$ where $J=\overline{\bigcup_{i=1}^{\infty} f^{i}(\operatorname{conv}(\operatorname{orb}(p)))}$.

Proof. Assume that $c \notin \operatorname{conv}(\operatorname{orb}(p))$ and $c \in J$. Then $\operatorname{per}(p)=m>1$. Let $a=\min (\operatorname{orb}(p))$ and $b=\max (\operatorname{orb}(p))$. We can assume that $c>b$. Because $c \in J$, there is $d \in(a, b)$ and $k \in \mathbb{N}$ such that $f^{k+i}(d)>b$ for $i \in\{1, \ldots, m\}$. But there are $i, j \in\{1, \ldots, m\}$ such that $f^{k+i}(a)=a$ and $f^{k+j}(b)=a$. Hence $[a, b] \subset f^{k+i}([a, d]) \cap f^{k+j}([d, b])$, and we have that $E(f)>0$ - a contradiction. 
Lemma 3.3. Let $f \in C^{0}(I, I)$ and $E(f)=0$. Then for all $n \in\{0,1, \ldots\}$ and $p \in \operatorname{Per}(f)$ such that $f^{2^{n}}(p) \neq p$ there is a closed periodic interval $J \subset I$ with period $2^{n}$ such that $p \in J$.

Proof. If $n=0$ then we can take $J=\bigcap_{i=1}^{\infty} f^{i}(I)$. Now we use induction. Let Lemma 3.3 hold for $n$. We will prove it for $n+1$.

Let $p \in \operatorname{Per}(f)$ and $f^{2^{n+1}}(p) \neq p$. From [2, Lemma 8] we have that $\operatorname{orb}(p)=$ $S_{1} \cup S_{2}$ where $S_{1}<c<S_{2}, c \in \operatorname{Fix}(f), f\left(S_{1}\right)=S_{2}, f\left(S_{2}\right)=S_{1}$, and $S_{1}, S_{2}$ are periodic orbits for $g=f^{2}$ with period $\frac{\operatorname{per}(p)}{2}>2^{n}$.

Let $J_{1}=\overline{\bigcup_{i=1}^{\infty} f^{2 i}\left(\operatorname{conv}\left(S_{1}\right)\right)}$ and $J_{2}=\overline{\bigcup_{i=1}^{\infty} f^{2 i}\left(\operatorname{conv}\left(S_{2}\right)\right)}$. We have that $f\left(J_{1}\right)=J_{2}$ and $f\left(J_{2}\right)=J_{1}$.

If, for example, $p \in S_{1}$, then $p \in \operatorname{Per}\left(\left.g\right|_{J_{1}}\right)$ and $f^{2^{n}}(p) \neq p$. Hence there is closed periodic interval $J \subset J_{1}$ for function $g$ such that $\operatorname{per}(J)=2^{n}$ and $p \in J$. But from Lemma 3.2 we have $J_{1} \cap J_{2}=\varnothing \quad\left(c \notin J_{1} \cup J_{2}\right)$, and from here it is easy to see that $J$ is a periodic interval for function $f$ with period $2^{n+1}$.

Lemma 3.4. Let $f \in C^{0}(I, I)$ and $E(f)=0$. Let $\left\{J_{k}\right\}_{k \in K}$ be a set of periodic intervals with period $2^{m}$, and let $J=\bigcup_{k \in K} J_{k}$ be an interval. Then $J$ is a periodic interval with period $2^{m-1}$ or $2^{m}$.

Proof. We have that $f^{2^{m}}(J)=\overline{\bigcup_{k \in K} f^{2^{m}}\left(J_{k}\right)}=\overline{\bigcup_{k \in K} J_{k}}=J$, and hence it suffices to show that if $f^{2^{m-2}}(p)=p$ then $p \notin J$.

Assume that $p \in J$ and $f^{2^{m-2}}(p)=p$.

The set $P=\left\{x \in I ; f^{2^{m-1}}(x)=x\right\}$ is closed and hence from the construction of $J$ :

- there is an interval $J^{*}=[p, q]^{*} \subset J ; p \neq q$ such that $P \cap(p, q)^{*}=\varnothing$ and we can take for $J^{*}$ the maximal of such intervals, or

- there is an interval $J^{*}=\left[q_{1}, q_{2}\right] \subset J ; q_{1} \neq q_{2}$ such that $P \cap\left[q_{1}, q_{2}\right]=$ $\left\{q_{1}, q_{2}\right\}$.

In both cases, $J^{*}=\overline{\bigcup_{k \in K^{*}} J_{k}}$ where $K^{*} \subset K$ and $f^{2^{m-1}}\left(J^{*}\right) \cap J^{*} \neq \varnothing$. Because $f^{2^{m}}\left(J^{*}\right)=J^{*}$, it follows that $f^{2^{m-1}}\left(J^{*}\right)=J^{*}$ and for $k \in K^{*}$ we have $\left(J_{k} \cup\right.$ $\left.f^{2^{m-1}}\left(J_{k}\right)\right) \subset J^{*}$. Set $J_{k}^{*}=\operatorname{conv}\left(J_{k} \cup f^{2^{m-1}}\left(J_{k}\right)\right)$. We have that $f^{2^{m-1}}\left(J_{k}^{*}\right) \supset J_{k}^{*}$ and hence there is $x \in J_{k}^{*}$ such that $f^{2^{m-1}}(x)=x$. But $x \notin J_{k} \cup f^{2^{m-1}}\left(J_{k}\right)$ and hence $x \in \operatorname{int}\left(J_{k}^{*}\right) \subset \operatorname{int}\left(J^{*}\right)$-a contradiction to the construction of $J^{*}$.

Lemma 3.5. Let $f \in C^{0}(I, I)$ and $E(f)=0$. Then the following conditions hold:

(i) There is exactly one cover system for $f,\left\{M_{i}\right\}_{i=0}^{\infty}$.

(ii) $f\left(M_{i}\right)=M_{i}$.

(iii) $M_{i+1} \subset M_{i}$ for $i \in\{0,1, \ldots\}$.

(iv) We can say that $M_{i}=\bigcup_{k \in \mathbb{R}_{i}} J_{k}^{i}$ where $\mathbb{R}_{i} \subset \mathbb{R}, J_{k_{1}}^{i} \cap J_{k_{2}}^{i}=\varnothing$ for $k_{1} \neq k_{2}$, $J_{k}^{i} \subset I$ is a closed interval, and for any interval $J \subset M_{i}$ there is $k \in \mathbb{R}_{i}$ such that $J \subset J_{k}^{i}$. Now if $\left|J_{k}^{i}\right|>0$ then $J_{k}^{i}$ is a periodic interval with period $2^{i}$ or $2^{i-1}$.

Proof. (i) Let $p \in \operatorname{Per}(f)$ and $i \in\{0,1,2, \ldots\}$. If $f^{2^{i}}(p)=p$ then let $I_{p}^{i}=\varnothing$ else let $I_{p}^{i}$ be the minimal closed periodic interval with period $2^{i}$ such 
that $p \in I_{p}^{i}$. The existence of $I_{p}^{i}$ follows from Lemma 3.3 ( $I_{p}^{i}$ is the intersection of all closed $2^{i}$-periodic intervals which contain $p$ ). Now let $M_{i}=\overline{\bigcup_{p \in \operatorname{Per}(f)} I_{p}^{i}}$ and it is easy to see that $\left\{M_{i}\right\}_{i=0}^{\infty}$ is a unique (from minimality) cover system.

(ii) It is easy to see that $f\left(I_{p}^{i}\right)=I_{f(p)}^{i}$ for any $p \in \operatorname{Per}(f)$ and $i \in\{0,1, \ldots\}$. Now because $f(\operatorname{Per}(f))=\operatorname{Per}(f)$, we have that $f\left(M_{i}\right)=M_{i}$. $M_{i}$.

(iii) From the proof of Lemma 3.3 we have that $I_{p}^{i+1} \subset I_{p}^{i}$ and hence $M_{i+1} \subset$

(iv) The first part is only the decomposition of a closed subset of a real interval into closed connected subsets, and the second part follows from Lemma 3.4 .

Lemma 3.6. Let $f \in C^{0}(I, I), E(f)=0$, and $\left\{M_{i}\right\}_{i=0}^{\infty}$ be a cover system for $f$, where $M_{i}=\bigcup_{k \in \mathbb{R}_{i}} J_{k}^{i}$ as in Lemma 3.5. Then if $J_{k}^{0}=[a, b]$ where $a<b$, then $f(a) \neq a$ and $f(b) \neq b$.

Proof. Let $P=\left\{p \in \operatorname{Per}(f) \cap J_{k}^{0} ; f(p) \neq p\right\} \quad(P \neq \varnothing$ from minimality of $\left.M_{0}\right)$ and $J_{p}=\overline{\bigcup_{i=1}^{\infty} f^{i}(\operatorname{conv}(\operatorname{orb}(p)))}$ for $p \in P$. Let $p_{0}, p \in P$. We have that $J_{p_{0}} \cap \operatorname{orb}(p)=\varnothing$ or $J_{p} \subset J_{p_{0}}$.

If $\operatorname{conv}(\operatorname{orb}(p)) \cap J_{p_{0}}=\varnothing$ then there is a fixed point between $\operatorname{conv}(\operatorname{orb}(p))$ and $J_{p_{0}}$ (because

$$
f(\max (\operatorname{orb}(p)))<\max (\operatorname{orb}(p)), \quad f(\min (\operatorname{orb}(p)))>\min (\operatorname{orb}(p)),
$$

and Lemma 3.2) and hence $J_{p_{0}} \cap J_{p}=\varnothing$.

If $J_{p_{0}} \cap J_{p} \neq \varnothing$ and $\left|J_{p}\right|>\left|J_{p_{0}}\right|$ then $J_{p_{0}} \subset \operatorname{conv}(\operatorname{orb}(p))$. So we have that $\operatorname{orb}(p)=S_{1} \cup S_{2}$ where $S_{1}<J_{p_{0}}<S_{2}$, and because there is a fixed point in $J_{p_{0}}$ from [2, Lemma 8] we have that $f\left(S_{1}\right)=S_{2}$ and $f\left(S_{2}\right)=S_{1}$. Hence it is easy to see that if there is a sequence $\left\{p_{i}\right\}_{i=0}^{\infty}, p_{i} \in P$, such that $J_{p_{i}} \subset J_{p_{i+1}}$ and $\left|J_{p_{i}}\right|<\left|J_{p_{i+1}}\right|$ then $\lim _{i \rightarrow \infty} J_{p_{i}}=\left[a^{*}, b^{*}\right]$ where $f\left(a^{*}\right)=b^{*}$ and $f\left(b^{*}\right)=a^{*}$.

Hence we can assume that $p_{0} \in P$ such that for any $p \in P$ either $J_{p} \subset J_{p_{0}}$ or $J_{p} \cap J_{p_{0}}=\varnothing$. Now if $J_{k}^{0}=J_{p_{0}}$ then we are done (see Lemma 3.2). So we can assume that $J_{p_{0}}=\left[a_{0}, b_{0}\right]$ and $a<a_{0}$. Let $J^{*}=\overline{\bigcup_{p \in P} J_{p}}$. If $P \cap\left[a, a_{0}\right)=\varnothing$ then $J^{*} \cap\left[a, a_{0}\right)=\varnothing$. If $P \cap\left[a, a_{0}\right) \neq \varnothing$ then there is a fixed point $c \in\left(a, a_{0}\right)$ (the same arguments as above) and we can assume that it is maximal. Then $P \cap\left[c, a_{0}\right)=\varnothing$ and hence (Lemma 3.2) $J^{*} \cap\left(c, a_{0}\right)=\varnothing$. So in both cases we can replace $J_{k}^{0}$ by $J^{*}$, which is a contradiction to minimality of $M_{0}$.

\section{REDUCTION FUNCTIONS}

Definition 4.1. Let $I=[a, b]$ be an interval and $\mathscr{A}=\bigcup_{i \in K} I_{i}$ where $I_{i} \subset I$ is a closed interval and $I_{i} \cap I_{j}=\varnothing$ for all $i \neq j$. We will call a function $h \in C^{0}(I, I)$ a reduction function for $\mathscr{A}$ if

$$
h(x)=x-\left|[a, x] \cap I_{j_{x}}\right|-\sum_{I_{i} \subset[a, x]}\left|I_{i}\right| \quad \text { where } x \in \operatorname{int}\left(I_{j_{x}}\right)
$$

(semihomeomorphism $h$ reduces the intervals $I_{i}$ to the points).

Lemma 4.2. Let $f \in C^{0}(I, I)$ be a nondegenerate function, $E(f)=0,\left\{M_{i}\right\}_{i=0}^{\infty}$ be a cover system for $f$, where $M_{i}=\bigcup_{k \in \mathbf{R}_{i}} J_{k}^{i}$ as in Lemma 3.5, $h$ be a 
reduction function for $M_{i}$, and $J=h(I)$. Then there is $g \in C^{0}(J, J)$ such that $h \circ f=g \circ h$ and $g$ is a nondegenerate function.

Proof. The existence of a $g \in C^{0}(J, J)$ such that $h \circ f=g \circ h$ follows from Lemma 3.5.

We can assume that $i=0$. If not, we only replace function $f$ by $f^{2^{i}}$ and $g$ by $g^{2^{i}}$ (it is easy to see that $g$ is nondegenerate if and only if $g^{2^{i}}$ is).

Let $J_{k}^{0}=[a, b]$, where $a<b$. Lemma 3.6 implies that there are $a^{*}, b^{*} \in I$ such that $a^{*}<a, b<b^{*}$, and if $x \in\left[a^{*}, a\right]\left(x \in\left[b, b^{*}\right]\right)$ then $f(x) \geq a$ $(f(x) \leq b)$. Hence if $x \in h\left(\left[a^{*}, a\right]\right) \quad\left(x \in h\left(\left[b, b^{*}\right]\right)\right)$ then $g(x) \geq h([a, b])$ $(g(x) \leq h([a, b]))$. But we have that $\operatorname{Per}(g)=\operatorname{Fix}(g)$ and $\left|h\left(\left[a^{*}, a\right]\right)\right| \neq 0 \neq$ $\left|h\left(\left[b, b^{*}\right]\right)\right|$ (see Lemma 3.5). Hence we have that

$$
\text { if }\left|J_{k}^{0}\right|>0 \text { then there is no } g \text {-nontrapping neighborhood of } h\left(J_{k}^{0}\right) \text {. }
$$

Let $x \in J, O_{x}$ be a neighborhood of $x, p \in \operatorname{Per}(g),[p, q]^{*}$ be an $m-g$ nontrapping neighborhood of $p, \lim _{n \rightarrow \infty} g^{m n}(x)=p$, and $z_{1}, z_{2} \in(p, q)^{*}$.

We will show that $m=1$. Let, for example, $p<q$. If $[p, q]$ is not a $g$-nontrapping neighborhood of $p$, then there is $z \in[p, q]$ such that for all $x \in[p, z]$ we have $g(x)<z$. Now from [14, Theorem 1] $g^{m}(x)<z$ for all $m \in \mathbb{N}$ and $x \in[p, z]$. Hence $[p, q]$ is not an $m$ - $g$-nontrapping neighborhood of $p$ for any $m \in \mathbb{N}$, which is a contradiction.

Hence we have that $m=1$ and from (6) that $h^{-1}(p)=p_{f} \in \operatorname{Fix}(f)$.

Let $h^{-1}\left([p, q]^{*}\right)=\left[p_{f}, q_{f}\right]^{*}$. We can assume that $p<q$ and hence $p_{f}<$ $q_{f}$. If $\left[p_{f}, q_{f}\right]$ is not an $f$-nontrapping neighborhood of $p_{f}$, then there are $z_{f}, z_{f}^{*} \in\left[p_{f}, q_{f}\right]$ such that $z_{f}^{*}<z_{f}$ and $f\left(\left[p_{f}, z_{f}\right]\right) \cap\left[p_{f}, z_{f}\right] \subset\left[p_{f}, z_{f}^{*}\right]$. Because $[p, q]$ is a $g$-nontrapping neighborhood of $p$, there is $J_{k}^{0}=[a, b] \subset$ $M_{0}$ such that $z_{f}, z_{f}^{*} \in J_{k}^{0}$. We have that $p_{f}<a$ and hence $f\left(\left[a, z_{f}\right]\right) \subset$ $\left[a, z_{f}^{*}\right]$, and similarly as in the proof of Lemma 3.6 we can replace $J_{k}^{0}$ by the set $J^{*}$ such that $J^{*} \cap\left(z_{f}^{*}, z_{f}\right)=\varnothing$, which is a contradiction to the minimality of $M_{0}$. Hence we have that $\left[p_{f}, q_{f}\right]^{*}$ is an $f$-nontrapping neighborhood of $p_{f}$.

Now let $x_{f} \in h^{-1}(x)$ and $z_{i_{f}} \in h^{-1}\left(z_{i}\right)$ for $i=1,2$.

We have that $z_{1_{f}}, z_{2_{f}} \in\left(p_{f}, q_{f}\right)^{*}, \lim _{n \rightarrow \infty} f^{n}\left(x_{f}\right)=p_{f}$, and there is a neighborhood $O_{x_{f}}$ of $x_{f}$ such that $h\left(O_{x_{f}}\right) \subset O_{x}$.

Because $f$ is nondegenerate, there is $n_{0} \in \mathbb{N}$ such that $\left[z_{1}, z_{2_{f}}\right] \subset f^{n_{0}}\left(O_{x_{f}}\right)$ and hence we have that $\left[z_{1}, z_{2}\right] \subset g^{n_{0}}\left(O_{x}\right)$.

Lemma 4.3. Let $f \in C^{0}(I, I)$ be a shrink function, $E(f)=0$, and $\left\{M_{i}\right\}_{i=0}^{\infty}$ be a cover system for $f$. Then for all $\varepsilon>0$ there is $\varepsilon^{*}>0$ and $i \in \mathbb{N}$ such that if $h_{i}$ is a reduction function for $M_{i}$ and $J \subset I$ is an interval with $|J|>\varepsilon$ then $\left|h_{i}(J)\right|>\varepsilon^{*}$. (In other words, $h_{i}^{-1}$ blows up small intervals to small intervals.)

Proof. Assume the contrary.

Let there be $\varepsilon>0$, an increasing sequence $\left\{n_{i}\right\}_{i=1}^{\infty}$, and a set of intervals $\left\{I_{i}\right\}_{i=1}^{\infty}$ such that $\left|I_{i}\right|>\varepsilon$ and $\left|h_{n_{i}}\left(I_{i}\right)\right| \leq \frac{1}{i}$ for all $i \in \mathbb{N}$.

We can assume that $\bigcap_{i=1}^{\infty} I_{i}=J$ such that $|J| \geq \frac{\varepsilon}{2}$ (if necessary we can consider proper subsequences). Hence $\lim _{i \rightarrow \infty}\left|h_{n_{i}}(J)\right|=0$, and from Lemma 3.5 we have $\left|h_{i}(A)\right| \geq\left|h_{j}(A)\right|$ for $i>j$ and $A \subset I$. Hence we have that $\left|h_{i}(J)\right|=0$ for $i \geq 0$ and so $J \subset M_{i}$ for $i \geq 0$. So there is a sequence $\left\{J_{k_{i}}^{i}\right\}_{i=0}^{\infty}$ such that 
$J \subset J_{k_{i}}^{i}$, and from Lemma 3.5 we obtain a contradiction to the assumption that $f$ is a shrink function.

\section{Proof of Main Theorem}

Theorem 5.1 (Gedeon and Kuchta [8]). Let $f \in C^{0}(I, I)$, and let there be an $m \in \mathbb{N}$ such that if $p \in \operatorname{Per}(f)$ then $f^{m}(p)=p$. Then $f$ has the shadowing property if and only if $f$ is a nondegenerate function.

Remark 5.2. This is a slight variation of [8, Theorem 2]. In [8] there is an assumption that $\operatorname{Per}(f)$ is nowhere dense in $I$ and there is a different definition of nondegenerate function.

But from [8, Lemma 7] it is easy to see that we can change the used definition of a nondegenerate function for Definition 1.4.

Moreover, if $\operatorname{Per}(f)$ is dense in some interval $J \subset I$ then we have $J \subset$ $\operatorname{Per}(f)$ (see [13, 4.1 Lemma]), and hence it is easy to see that $f$ is a degenerate function and does not have the shadowing property. So this assumption is not necessary.

Main Theorem. Let $f \in C^{0}(I, I)$ and $E(f)=0$. Then $f$ has the shadowing property if and only if $f$ is a nondegenerate shrink function.

Proof. $\Rightarrow$ If $f$ has the shadowing property then Propositions 2.2 and 2.3 imply that $f$ is a nondegenerate shrink function.

$\Leftarrow$ Let $f$ be a nondegenerate shrink function and $\varepsilon>0$. From Lemma 3.5 there is a cover system $\left\{M_{i}\right\}_{i=0}^{\infty}$ of $f$. From Lemma 4.3 there is $\varepsilon^{*}>0$ and $i \in \mathbb{N}$ such that if $h$ is a reduction function for $M_{i}$ and $x, y \in I$ then

$$
\text { if }|h(x)-h(y)|<\varepsilon^{*} \text { then }|x-y|<\varepsilon .
$$

Let $J=h(I)$. From Lemma 4.2 there is $g \in C^{0}(J, J)$ such that $h \circ f=g \circ h$ and $g$ is a nondegenerate function. From Theorem 5.1 we have that $g$ has the shadowing property. Hence there is $\delta>0$ such that every $\delta$-chain of $g$ is $\varepsilon^{*}$-shadowed by some $y^{*} \in J$.

Now let $\mathbf{X}_{\delta}=\left\{\mathbf{x}_{i}\right\}_{i=0}^{\infty}$ be a $\delta$-chain of $f$. Then $\mathbf{X}_{\delta}^{*}=\left\{h\left(\mathbf{x}_{i}\right)\right\}_{i=0}^{\infty}$ is a $\delta$-chain of $g$ and there is $y^{*} \in J$ such that $\left|g^{i}\left(y^{*}\right)-h\left(\mathbf{x}_{i}\right)\right|<\varepsilon^{*}$ for all $i \geq 0$.

Let $y \in h^{-1}\left(y^{*}\right)$. We have that $h\left(f^{i}(y)\right)=g^{i}(h(y))=g^{i}\left(y^{*}\right)$ and from (7) we have $\left|f^{i}(y)-\mathbf{x}_{i}\right|<\varepsilon$ for all $i \geq 0$.

\section{REFERENCES}

1. D. V. Anosov, On a class of invariant sets for smooth dynamical systems, Proc. 5th International Conf. on Nonlinear Oscillations 2, Math. Inst. Ukrainian Acad. Sci., Kiev, 1970, pp. 39-45. (Russian)

2. L. Block, Simple periodic orbits of mappings of the interval, Trans. Amer. Math. Soc. 254 (1979), 391-398.

3. R. Bowen, On axiom A diffeomorphisms, Regional Conf. Ser. in Math., vol. 35, Amer. Math. Soc., Providence, RI, 1978.

4. A. Boyarsky and P. Góra, The pseudo-orbit shadowing property for Markov operators in the space of probability density functions, preprint, 1989.

5. L. Chen, Linking and the shadowing property for piecewise monotone maps, Proc. Amer. Math. Soc. 113 (1991), 251-263. 
6. __ Shadowing property for nondegenerate zero entropy piecewise monotone maps, preprint, SUNY, Stony Brook, 1990.

7. E. M. Coven, I. Kan, and J. A. Yorke, Pseudo-orbit shadowing in the family of tent maps, Trans. Amer. Math. Soc. 308 (1988), 227-241.

8. T. Gedeon and M. Kuchta, Shadowing property of continuous maps, Proc. Amer. Math. Soc. 115 (1992), 271-281.

9. S. M. Hammel, J. A. Yorke, and C. Grebogi, Do numerical orbit of chaotic dynamical processes represent true orbits?, J. Complexity 3 (1987), 136-145.

10. I. Kan, Shadowing property of quadratic maps, preprint, 1989.

11. M. Kuchta, Characterization of chaos for continuous maps of the circle, Comment. Math. Univ. Carolin. 31 (1990), 383-390.

12. T. Pennings and J. Van Eeuwen, Pseudo-orbit shadowing on the unit interval, preprint, 1990.

13. D. Preiss and J. Smital, A characterization of nonchaotic continuous maps of the interval stable under small perturbations, Trans. Amer. Math. Soc. 313 (1989), 687-696.

14. A. N. Šarkovskiĭ, On cycles and the structure of a continuous mapping, Ukrain. Mat. Ž. 17 (1965), 104-111. (Russian)

Mathematical Institute, Slovak ACademy of Sciences, Štefánikova 49, 81473 BratiSlava, Czecho-Slovakia

Current address: Department of Mathematics, University of Warwick, Coventry, CV4 7AL, Great Britain

E-mail address: mk@maths.warwick.ac.uk 